\title{
Model for the Identification of Diseases in the Banana Plant Using a Convolutional Neural Network
}

\author{
Fredy H. Martínez S. ${ }^{1, *}$, Edwar Jacinto G. ${ }^{2}$ and Fernando Martínez S. ${ }^{3}$

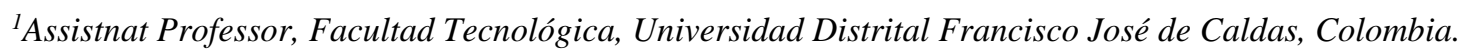 \\ ORCID: 0000-0002-7258-3909 \\ ${ }^{2}$ Assistnat Professor, Facultad Tecnológica, Universidad Distrital Francisco José de Caldas, Colombia. \\ ORCID: 0000-0003-4038-8137 \\ ${ }^{3}$ Assistnat Professor, Facultad Tecnológica, Universidad Distrital Francisco José de Caldas, Colombia. \\ ORCID: 0000-0003-2895-3084
}

\begin{abstract}
Banana cultivation is an important source of economic income for several developing countries, with economies heavily dependent on their agricultural production. Such is the case of Colombia, a banana export country in which much of its production is still carried out by small peasant families without a high degree of modernization. The plant is quite sensitive to diseases caused by environmental conditions, bacteria, and viruses. Infections can spread rapidly and cause great damage to the plantations. For this reason, it is necessary to develop high performance and very low-cost technologies capable of quickly identifying the damage, to control it and reduce losses. In this article, we propose a convolutional model based on a deep neural network for the classification of banana plant leaf damage from images. The model is trained specifically for this problem with real images captured in different states of affectation of leaves of the plant. The model is suitable to be propagated on a very low-cost embedded system, and therefore suitable to be used in small plantations. The performance of the model demonstrates a high capacity to differentiate the damage to the leaves and the cause of it, making it possible to quickly formulate a treatment strategy.
\end{abstract}

Keywords: Bacterial Wilt, Banana, Black Sigatoka, Deep Neural Network, Image Processing.

\section{INTRODUCTION}

The banana is known by the scientific name of the Muse of Paradise. It is a tropical plant cultivated in warm and humid regions within developing countries, and in Colombia, it constitutes an important part of its production and export income [1, 2]. It is normally cultivated by small farming families, and only about $15 \%$ is used for international trade. Latin America and the Caribbean account for 80 percent of exports with approximately three million tons [1]. Even so, at the global level, these crops occupy the 12th place for the economy of crops and the amount of production [3]. Colombia is the fourth-largest supplier of bananas in the world (1806 tons in 2019) [4].

In addition to human and animal food, the banana is used in studies for implementation in different fields because of its different properties. One of the alternatives uses is characterized by the extraction of starch from banana waste, used to make the dosage of thermoplastic polymers [5]. Bananas are also used in a fermentation process combined with different ingredients, to be used in the feeding of recently castrated and weaned pigs. Studies are being carried out in the field of environmentally friendly materials, including the modification of cellulose taken from banana fiber to make new biopolymers with great mechanical and chemical properties, to obtain a more degradable and environmentally friendly material [6]. Another case that can be mentioned is the use of banana stem to make ecological molded pulp, forming a favorable alternative source for this production [7].

Different diseases attack this crop, and that requires quick and effective action to control their spread and the consequent economic damage. One of them is the lack of magnesium, the lack of this nutrient prevents the proper growth of the plant, which also affects the reduction of glucose, a key element in the fruit of the plant [8]. Another disease that affects the growth of the banana plant is the banana streak virus, which can be identified from the fifth month of the plant when the leaf begins to show a change in color to a reddish yellow [9].

Another very common disease is Black Sigatoka, caused mostly by the agent Mycosphaerella Fijiensis, and its teleomorphs. It is a disease considered to be the cause of great damage to crops, and a great deterioration of the leaf area. Besides, it affects the growth and productivity of plants by reducing the capacity for photosynthesis when not controlled. When a plant has Pseudocercospora Fijiensis (Teleomorph Mycosphaerella Fijiensis) its fruit is also infected, so the export of this fruit can bring the disease to countries that are not infected [10]. Black Sigatoka has six stages of maturity, the first stage being the mildest and the sixth being the strongest of the disease [11].

\footnotetext{
* Corresponding Author
} 
International Journal of Engineering Research and Technology. ISSN 0974-3154, Volume 13, Number 10 (2020), pp. 2668-2673

(C) International Research Publication House. https://dx.doi.org/10.37624/IJERT/13.10.2020.2668-2673

One way to detect the severity of the disease in plants is through a geostatistical study, taking the youngest leaf of the infected plant as an indicator variable [12].

The Timorex Gold method is a way to combat Sigatoka. It is based on the extract of Australian tea tree essential oil, which has a high efficiency if used between stages one to four [13]. Another way to combat this disease is by using bio fungicides based on citronella and mancozeb essential oils [14]. Ozone dissolved in water can also be used for the control of this disease [15]. Another way to decrease the great damage of the disease and increase the production is to control the level of shade by $20 \%$ [16].

Another banana leaf disease is withering due to bacteria. There are two kinds of bacteria, the first one is Xanthomonas, which is a genus of proteobacteria (containing a large variety of pathogens) that has attacked all banana crops in Africa for many years, being a major problem in the economy of small farmers. It also generates health problems in people who consume it [17]. The ABB Muse in northern Mozambique is highly sensitive to the spread of this disease [18]. The second bacterium is Fusarium. The disease is identified as the major threat to banana exports and belongs to a family of filamentous fungi that carry approximately 117 bacteria belonging to 20 different families [19]. This bacterium, normally distributed in the soil, produces chlamydospores (thick-walled spores) capable of surviving in the soil for more than 20 years. Still, very little is known about this disease [20].

There are different methods to detect banana plant leaf diseases. One of them is the acquisition of high-resolution visible color images, another corresponds to the combination of these images with low-resolution hyperspectral images to obtain a better quality and color HS image. These images were used as control points and multisensorial data recording in some investigations [21]. For the identification of the bananascratch virus, an image of a healthy leaf processed by an algorithm was used, taking the number of pixels of this image as a threshold to make the comparison of pixels against another processed image. If the pixels of the image pass the threshold, the presence of the virus in the leaf is detected [9]. Image processing along with segmentation, labeling, and filtering are used to determine if a banana leaf is good or defective, classifying with numbers the defects such as pus, spider webs, tears among others. Extracting color, characteristics, and estimating boundary lengths [22].

The image pre-processing strategies are very robust to identify and isolate areas of interest in the leaves. However, strategies for efficient and reliable damage classification are still in strong development. In this sense, we propose the development of a low cost and high-performance system for the classification of these images by using a deep neural model trained for this problem [23, 24]. The final objective is to develop a low-cost embedded system capable of identifying characteristics in banana leaves that will allow the diagnosis of possible damage to the plant, particularly for diseases such as Black Sigatoka and Fusarium [25].

The following part of the paper is arranged in this way. Section II presents preliminary concepts and problem formulation. Section III illustrates the design profile and development methodology. Section IV we present the preliminary results. And finally, in Section V, we present our conclusions.

\section{PROBLEM FORMULATION}

The problem to be solved consists in the design of a model for the classification of banana leaf diseases based on color images of the plants. This model must operate in real-time, and have a high performance in the classification. Besides, it is expected that the prototype can be implemented on an embedded system, which is why it is essential to restrict the processing and storage requirements to the maximum.

The diseases to be categorized are Bacterial Wilt and Black Sigatoka. Also, it is necessary to differentiate the healthy leaves from the sick ones. Therefore, the model must contain three categories, which have been labeled as follows:

- bbs: For banana leaves sick with Black Sigatoka.

- bbw: For banana leaves diseased with Bacterial Wilt.

- healthy: For healthy banana leaves.

The images are to be pre-processed before being submitted to the classification model. This pre-processing consists of image segmentation and labeling to remove the background and retain only the ROI (Region of Interest), i.e. the banana leaf. Color level adjustment filters are also applied. This ensures that the model will identify and learn only the characteristics of the banana leaf and its diseases. The same treatment should be applied to unknown images that are intended to be propagated and classified with the model.

For the architecture of the deep neuronal model, the use of a DenseNet (Dense Convolutional Network) type convolutional network is proposed. In this network, as in any convolutional network, convolution layers with specially designed kernels are used to identify characteristics in the images. However, unlike other convolutional networks, its dense structure leads to a lower amount of parameters compared to others like ResNet (Residual Neural Network), without sacrificing performance. This is because its design is based on the concept that the accuracy of the network increases if it contains shorter connections between the layers near the input and the layers near the output. Fig. 1 shows a section of this architecture where it is detailed how the leap forward in the layers is made.

\section{METHODOLOGY}

The goal is to get a model to run on an embedded system. In that sense, we selected as hardware the DragonBoard 410c from Arrow Electronics. This board has a Qualcomm APQ8016e 64-bit quad-core processor, Wi-Fi, Bluetooth, and GPS connectivity, and support for Windows 10 IoT Core, Android 5.1, and Debian 8.0. On this card, we installed Keras and Tensorflow over Debian as software support to propagate the neural model.

The dataset was built with 200 images in each category, for a total of 600 images (Fig. 2). These images were taken from real plants and classified by experts. These images were randomly 
International Journal of Engineering Research and Technology. ISSN 0974-3154, Volume 13, Number 10 (2020), pp. 2668-2673

(C) International Research Publication House. https://dx.doi.org/10.37624/IJERT/13.10.2020.2668-2673

mixed in the list to improve the performance of the network. Besides, all were resized to a size of $400 \times 400$ pixels in RGB format (all three color matrices were kept). The aspect ratio of the images was not considered important when resizing them, since it is easier for the network to work with images of the same size, and the characteristics sought in the images are not lost when changing the aspect ratio (Fig. 3).

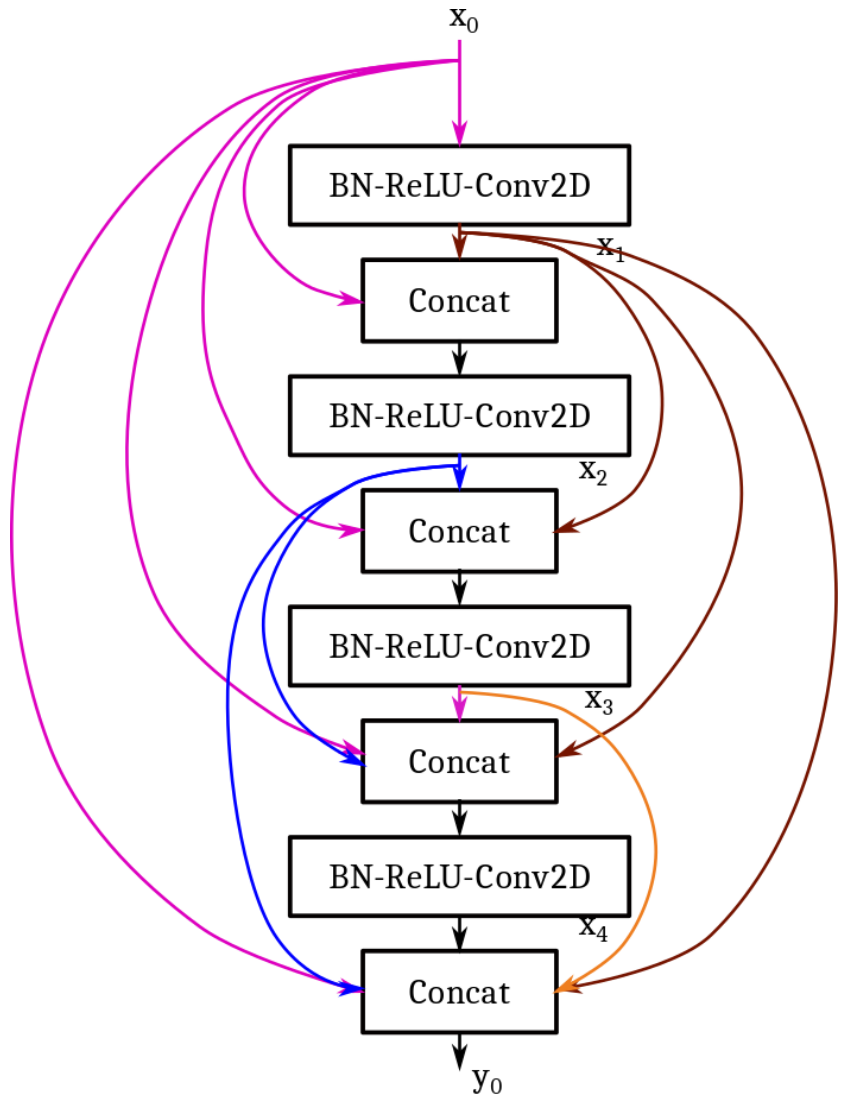

Fig. 1. Densely Connected Convolutional Network (DenseNet) architecture

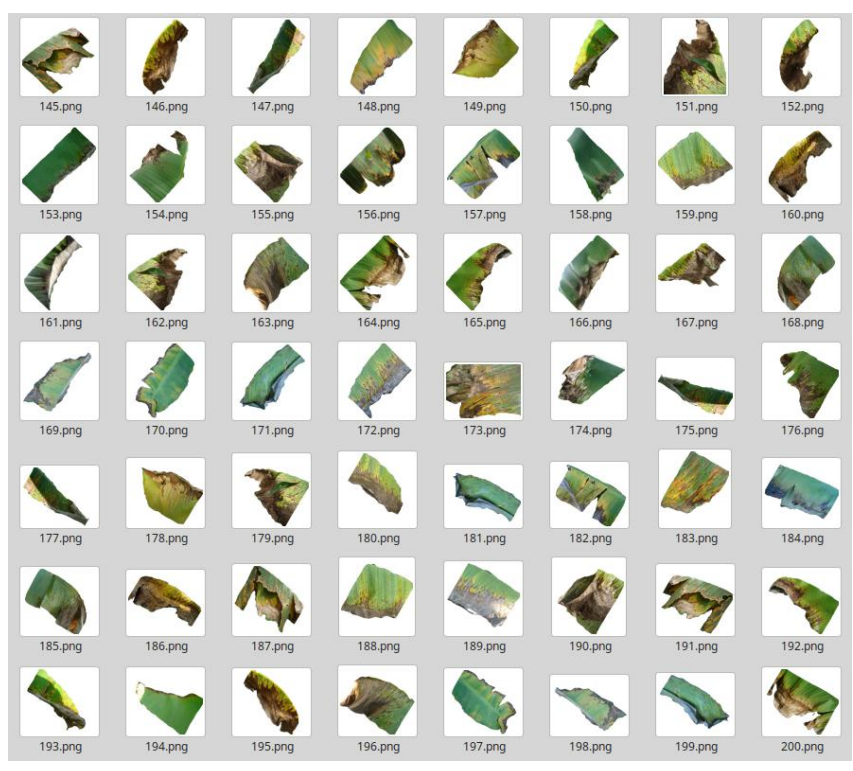

Fig. 2. Sample of the dataset corresponding to the category bbs (Banana Black Sigatoka)



Fig. 3. Image of the dataset after segmentation, labeling, filtering and scaling

The color matrices of the images were normalized to values between 0 and 1 corresponding to the color depth. The dataset was randomly separated into two groups, $80 \%$ of the images (480 images) were used for training, and the remaining $20 \%$ (120 images) for model validation (images unknown to the trained model). In the network structure, the number of nodes in the input layer is defined according to the size of the images, in this case, $400 * 400 * 3=480,000$ input nodes. The output nodes are defined by the number of categories, in our case three, one-hot coding was used on the output labels to establish this structure. The network has a total of 7,040,579 parameters, of which 6,956,931 were adjusted during training (Fig. 4).

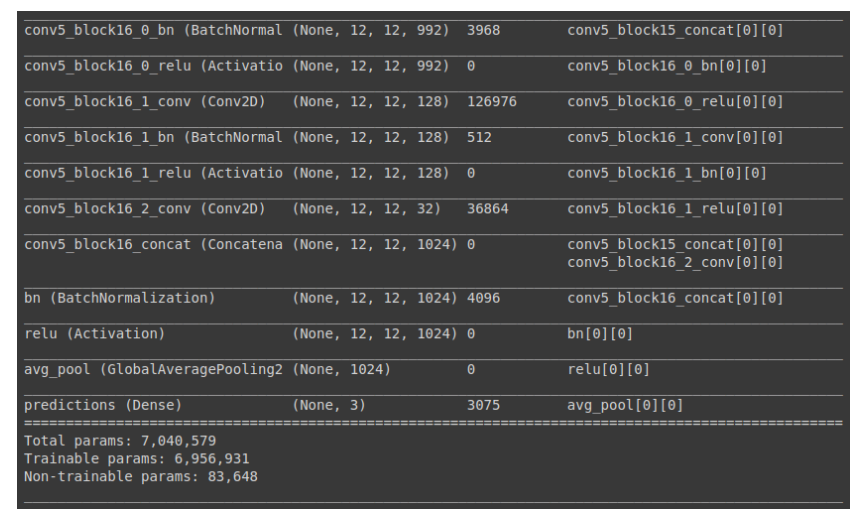

Fig. 4. Detail of network parameters and structure of its last layers

The network was created with the input and output sizes described above and without the use of pre-trained weights. The network architecture is made up of 121 layers. These layers are distributed in a $5+(6+12+24+16) * 2=121$ structure, where 5 is (convolutional, pooling) +3 transition layers + classification layer. Multiplication by 2 is done because each dense block has two layers ( $1 \times 1$ convolutional and $3 \times 3$ convolutional). The model was compiled using the stochastic gradient descent 
International Journal of Engineering Research and Technology. ISSN 0974-3154, Volume 13, Number 10 (2020), pp. 2668-2673

(C) International Research Publication House. https://dx.doi.org/10.37624/IJERT/13.10.2020.2668-2673

function as an optimization function, the categorical crossentropy function as an error measure, and the accuracy (or hit rate) and MSE (mean quadratic errors) metrics to evaluate the behavior of the network throughout each epoch.

The training was conducted over 30 epochs with a batch size of 32. Throughout the training, the accuracy increased from $83.8 \%$ to $92.5 \%$ for the training data. The final accuracy for the validation data was $72.5 \%$.

\section{RESULTS AND DISCUSSION}

Fig. 5 shows the behavior of the trained model. The error produced by the training data is continuously reduced during the first 20 epochs. During the last 10 epochs, the reduction is so low that it can be considered constant and indicates that it is not necessary to increase the training epochs. The error produced by the validation data is also reduced continuously throughout the training, but its value is always higher than that achieved by the training data ( 0.8 versus 0.2$)$. The accuracy of the training data increased sharply during the first five epochs, and then more smoothly during the rest of the training until it almost saturated its value at the end of the 30 epochs. On the other hand, the validation data showed a continuous increase in accuracy throughout the training process, approaching the training data values, and suggesting that it may even increase with a greater number of epochs.

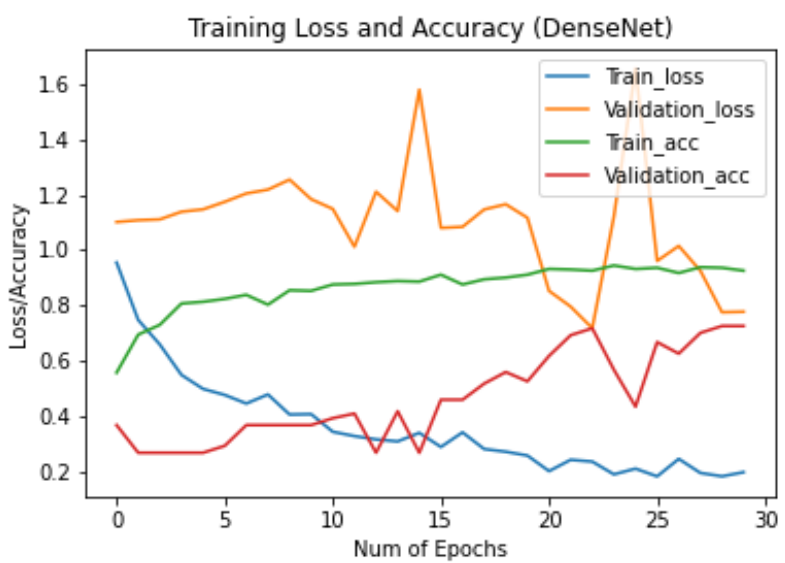

Fig. 5. Model behavior based on training and validation data

For a more complete evaluation of the model's performance we calculate, in addition to accuracy, the metrics of recall, f1score, and support (Fig. 6). The average accuracy value for the three categories (73\%), as well as the individual values per category, confirm the results in Fig. 5. The recall and f1-score corroborate the results both per category and average (72\%). We also calculated the confusion matrix and the ROC curve of the network (Figs. 7, 8, and 9). The best behavior is had for the first category, of the 44 leaves sick with Black Sigatoka the model correctly classified 35 (79.5\%). The nine incorrectly classified leaves were categorized as diseased with Bacterial Wilt. This result is quite good in principle, even more so considering that no leaves in this category were classified as healthy.

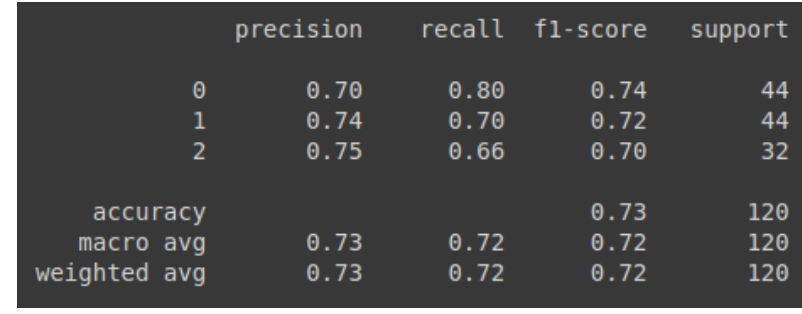

Fig. 6. Model metrics



Fig. 7. Confusion matrix

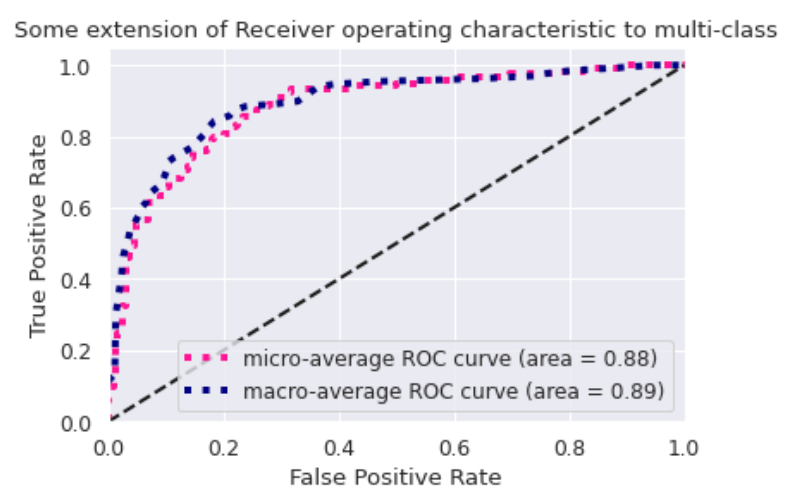

Fig. 8. ROC curve (average behavior)

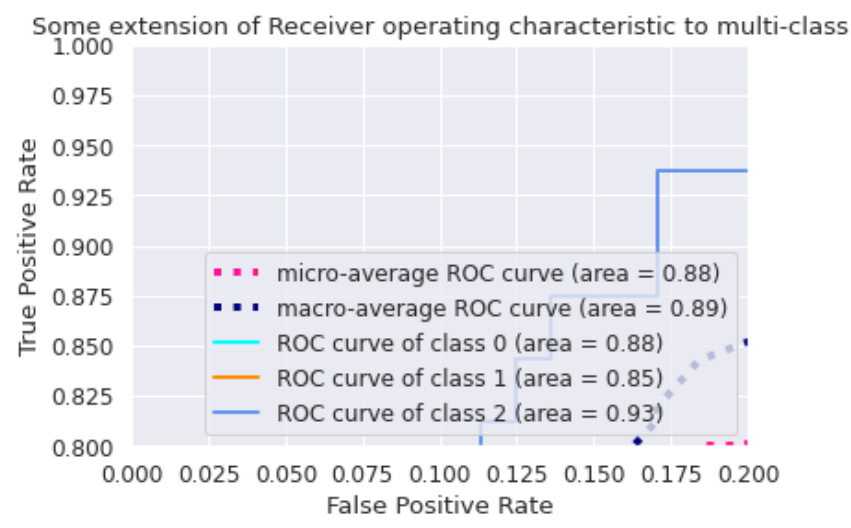

Fig. 9. ROC curve (behavior by class) 
The second category performed poorly, and even quite acceptably. Of the 44 leaves belonging to the second category, 31 were correctly categorized as having Bacterial Wilt disease $(70.4 \%)$. Of the remaining leaves, half were mistaken for Black Sigatoka and the other half for healthy leaves. Finally, of the 44 healthy leaves unknown to the model, 21 were correctly categorized $(47.8 \%)$, nine were confused as sick with Black Sigatoka, and two as sick with Bacterial Wilt.

The ROC curve shows that the average behavior of the three categories is very similar. By adjusting the thresholds between categories it is possible to increase the true positive rate above $80 \%$ in all three cases, with particular benefit in the category corresponding to healthy banana leaves. In principle, this is a good model for rapid leaf sorting from images, which can be used with different images of the crop taken with a commonly used smartphone.

\section{CONCLUSION}

This paper presents the design of a model based on convolutional neural networks for the rapid and safe classification of diseases in the banana plant leaf. Banana is a very important export product in Colombia, as well as for other countries with tropical climates, however, its cultivation is carried out by small families with little access to modern cultivation techniques. As a consequence, when there are outbreaks of diseases in the plant, these spread rapidly with a strong economic impact, both at the social level in the communities, as well as at the level of economic income of the country. Among the solutions proposed for rapid action against the advance of these diseases, the development of an autonomous and very low-cost system capable of identifying plant diseases from images of their leaves is proposed. For the development of the prototype, Black Sigatoka and Bacterial Wilt were selected as diseases to be identified. These two diseases produce visually identifiable damage on the leaves of the plant, making it feasible to use a classification model developed for images. DenseNet (Dense Convolutional Network) was selected as the model architecture due to its high comparative performance and reduced size of the parameters to be trained. The topology was adjusted to $400 * 400$ pixels color images in RGB format, and three output categories, adding the classification as a healthy leaf to the two selected diseases. 200 real images per category were used to ensure a balance between the categories. The training was carried out over 30 epochs taking care not to over-adjust the network. To fine-tune the parameters, the error was evaluated using the categorical crossentropy function, and optimized using the stochastic gradient descent function. The final accuracy achieved by the model was $92.5 \%$ for the training data and $72.5 \%$ for the validation data (images unknown to the model). This model was implemented on a DragonBoard 410c from Arrow Electronics, running a Debian OS. Preliminary results show low resource consumption, low cost, and acceptable performance for realworld implementation.

\section{ACKNOWLEDGMENTS}

This work was supported by the Universidad Distrital Francisco José de Caldas, specifically by the Technological Faculty. The views expressed in this paper are not necessarily endorsed by Universidad Distrital. The authors thank all the students and researchers of the research group ARMOS for their support in the development of this work.

\section{REFERENCES}

[1] FAO, "Enfoque de productos básicos", 2019.

[2] V. Guerrero and W. Sánchez, "Ecology, construction, and innovation: An alert towards change", Tekhnê, vol. 15, no. 2, pp. 45-58, ISSN 1692-8407.

[3] A. Benavides, "Enfermedades causadas por bacterias fitopatogenas en cultivos de banano (musa aaa) y plátano (musa abb) y sus estrategias de control", 2019.

[4] FAO, "Análisis del mercado del banano. panorama general de febrero de 2020", 2019.

[5] J. Amaya, "Estudio de la dosificación del almidón extraído del banano en un polímero de tipo termoplástico", Revista Colombiana de Quimica, 2018.

[6] A. Pinos and J. Braulio, "Modification of the cellulose obtained from banana fiber for to use in biodegradable polymers", Afinidad, pp. 45-51, 2019.

[7] P. Rattanawongkun, N. Kunfoung, S. Klayya, T. Chotimarnon, S. Duangphet, N. Tawichai, U. Intatha, and N. Soykeabkaew, "Comparison of molded pulps from rice straw, pineapple leaf and banana stem pulps", ScienceAsia, vol. 46S, no. 1, p. 79, 2020, doi:10.2306/scienceasia1513-1874.2020.s011.

[8] H. Hongsu, "Changes of plant biomass partitioning, tissue nutrients and carbohydrates status in magnesiumdeficient banana seedlings and remedy potential by foliar application of magnesium", Phytochemistry, 2020.

[9] G. Karthik and N. Praburam, "Detection and prevention of banana leaf diseases from banana plant using embeeded linux board", Online International Conference on Green Engineering and Technologies (IC-GET 2016), IEEE, 2016, doi:10.1109/get.2016.7916817.

[10] R. Fullerton and S. Casonato, "The infection of the fruit of 'cavendish' banana by pseudocercospora fijiensis, cause of black leaf streak (black sigatoka)", European Journal of Plant Pathology, vol. 155, no. 3, pp. 779-787, jul 2019, doi:10.1007/s10658-019-01807-x.

[11] E. Alvarez, A. Pantoja, L. Ganan, and G. Ceballos, "La sigatoka negra en plátano y banano", Centro Internacional de Agricultura Tropical, 2013.

[12] J. Gomez, W. Torres, D. Cayon, L. Hoyos, and D. Castaneda, "Modelacion espacial de la sigatoka negra (mycosphaerella fijiensis m. morelet) en banano cv. gran enano", Revista Ceres, vol. 64, no. 1, pp. 47-54, feb 2017, doi:10.1590/0034-737x201764010007. 
[13] M. Reuveni, E. Sanches, and M. Barbier, "Curative and suppressive activities of essential tea tree oil against fungal plant pathogens", Agronomy, vol. 10, no. 4, p. 609, apr 2020, doi:10.3390/agronomy10040609.

[14] E. Jaramillo, "Efecto biofungicida de aceites esenciales en el control de la sigatoka negra (mycosphaerella fijiensis) en el cultivo de banano", 2017.

[15] A. Hidaldo, R. Castano, and C. Aguirre, "Relación de la concentración y frecuencia de aplicación de ozono con el nivel de daño de la sigatoka negra en banano. diseño de un protocolo de riego con agua ozonificada", Alternativas, 2015.

[16] J. Barrera, F. Barraza, and R. Campo, "Shadow effect on black sigatoka (mycosphaerella fijiensis morelet) in plantain cultivation cv harton (musa aab simmonds)", Revista U.D.C.A Actualidad \& Divulgación Científica, 2016.

[17] A. Geberewold, "Review on impact of banana bacterial wilt (xhantomonas campestris pv. musacerum) in east and central africa", Cogent Food \& Agriculture, vol. 5, no. 1, mar 2019, doi:10.1080/23311932.2019.1586075.

[18] W. Ocimati, G. Blomme, and H. Bouwmeester, "Dataset for mapping xanthomonas wilt of banana spread in african great lakes region and africa”, 2019, doi:10.7910/DVN/VHSOLX.

[19] M. Kaushal, R. Swennen, and G. Mahuku, "Unlocking the microbiome communities of banana (musa spp.) under disease stressed (fusarium wilt) and non-stressed conditions", Microorganisms, vol. 8, no. 3, p. 443, 2020, doi:10.3390/microorganisms8030443.

[20] M. Dita, B. Marcia, H. Daniel, E. Mizubuti, and P. Charles, "Fusarium wilt of banana: Current knowledge on epidemiology and research needs toward sustainable disease management", Frontiers in Plant Science, vol. 9, oct 2018, doi:10.3389/fpls.2018.01468.

[21] W. Liao, D. Ochoa, Y. Zhao, G. Villegas, and W. Philips, "Banana disease detection by fusion of close range hyperspectral image and high-resolution $\mathrm{rgb}$ image", IEEE International Geoscience and Remote Sensing Symposium IGARSS 2018, IEEE, 2018, doi:10.1109/igarss.2018.8519115.

[22] T. Dat, H. Nguyen, and T. Nguyen, "Detection and classification defects on exported banana leaves by computer vision", International Conference on System Science and Engineering (ICSSE 2019), IEEE, 2019, doi:10.1109/icsse.2019.8823097.

[23] F. Martinez, C. Hernández, and A. Rendón, “Identifier of human emotions based on convolutional neural network for assistant robot", TELKOMNIKA (Telecommunication Computing Electronics and Control), vol. 18, no. 3, p. 1499, jun 2020, doi:10.12928/telkomnika.v18i3.14777.

[24] F. Martinez, C. Hernández, and F. Martínez, "Evaluation of deep neural network architectures in the identification of bone fissures", TELKOMNIKA (Telecommunication Computing Electronics and Control), vol. 18, no. 2, p. 807, 2020, doi:10.12928/telkomnika.v18i2.14754.
[25] A. Cadena, E. Peña, and F. Martínez, "Especificación, diseño, montaje y evaluación de un quadrotor con sistema de comunicación inalámbrica bidireccional”, Tekhnê, vol. 13, no. 1, pp. 13-30, ISSN 1692-8407. 